RESEARCH NOTE

\section{Improvement and Application of a Polymerase Chain Reaction System for Detection of Wuchereria bancrofti in Culex quinquefasciatus and Human Blood Samples}

\section{André F Furtado $/{ }^{+}$, Frederico GC Abath, Lêda Regis, Yara M Gomes, Wagner A Lucena, Patricia B Furtado, Rafael Dhalia, Janaína C Miranda, Luc Nicolas*}

Centro de Pesquisas Aggeu Magalhães-FIOCRUZ, Caixa Postal 7472, 50670-420 Recife, PE, Brasil

*Institut de Recherches Médicales Louis Malardé, Papeete, Tahiti, French Polynesia

Key words: filariasis - Wuchereria bancrofti - Culex quinquefasciatus - Polymerase Chain Reaction

Wuchereria bancrofti, Brugia malayi, and Brugia timori are mosquito-transmitted nematodes that cause lymphatic filariasis in humans. Lymphatic filariasis is endemic over a wide geographic area covering Africa, Central and South America, Asia and Oceania. Recent surveys estimated that at least 120 millions are infected in the world, more than 90\% harbouring W. bancrofti (WHO 1994 TDR/CTD/FIL/Penang 1994/1, EA Ottensen et al. 1995 Parasitol Today 11: 129-31).

The traditional methods of detection of filarial parasites in humans (blood films or membrane filtration) or in mosquitoes (dissection and microscopic examination) are labour intensive, time-consuming and make species identification difficult.

This investigation received financial support from UNDP/World Bank/WHO, Special Programme for Research and Training in Tropical Diseases (TDR), Conselho Nacional de Pesquisa $(\mathrm{CNPq})$ and Fundação de Amparo à Ciência e Tecnologia de Pernambuco (FACEPE).

${ }^{+}$Corresponding author. Fax: +55-81-453.1911

Received 19 July 1996

Accepted 23 October 1996
Initial reports of Polymerase Chain Reaction (PCR) detection of $W$. bancrofti larvae in pools of Aedes polynesiensis, the mosquito vector of $W$. bancrofti in French Polynesia, have suggested the possibility that this technique may replace conventional detection methods in many settings ( $\mathrm{S}$ Chanteau et al. 1994 Trans R Soc Trop Med Hyg 88: 665-666).

Cloning and characterization of a 300 copy repeat sequence (the Ssp I repeat) in W. bancrofti [NV1 (5'-CGTGATGGCATCAAAGTAGCG-3') and NV 2 (5'-CCCTCACTTACCATAAGACAA$\left.3^{\prime}\right)$ ] have been previously described by M Zhong et al. 1996 (Am J Trop Med Hyg 54: 357-363) and DNA primers specific to the genus Wuchereria were designed for PCR (WHO 1993 TDR/WRKS/ FIL/93.1). In the present communication some applications of a PCR system using the same primers to detect $W$. bancrofti L3 in pools of Culex quinquefasciatus, by far the most predominant mosquito vector in the transmission of lymphatic filariasis, is presented. In addition, parasite DNA in sera of microfilaraemic patient can be detected using this technique.

W. bancrofti L3 larvae were obtained from laboratory reared $C$. quinquefasciatus fed on infected blood from patients in Recife, Brazil, with more than $1000 \mathrm{mf} / \mathrm{ml}$. Fourteen to 17 days after the blood meal the mosquitoes were dissected, larvae collected and transferred to microcentrifuge tubes. DNA extraction and PCR were performed on pools of 50 or 100 uninfected C. quinquefasciatus with a single L3 added. Geneclean (Bios 101) was used for final purification of DNA. DNA from mosquito pools spiked with L3 was suspended in $25 \mathrm{ml}$ of water. In preliminary experiments this material was serially diluted (1:5 up to $1: 15.625)$. The best amplification was consistently accomplished when $1 \mathrm{ml}$ of DNA diluted to 1:625 was used in the PCR. When $10 \mathrm{ml}$ out of $25 \mathrm{ml}$ of total PCR product was loaded on $1 \%$ ethidium bromide stained agarose gels, the expected $188 \mathrm{bp}$ product was observed. This PCR system can detect as little as $0.01 \mathrm{pg}$ of $W$. bancrofti DNA.

The assay is sensitive and reproducible, being able to detect a single L3 in all the pools of 50 (6/ 6 experiments) and 100 (10/10 experiments) whole mosquitoes (Fig.). This sensitivity in $C$. quinquefasciatus is apparently greater than that obtained for detection of $W$. bancrofti var pacifica in Aedes polynesiensis (L Nicolas et al. 1996 Trans $R$ Soc Trop Med Hyg 90: 136-9) where one L3 was detected only in 5 out of 10 pools of 100 whole mosquitoes.

PCR using sera from microfilaraemic patients, was also performed. Unprocessed serum gave identical results to phenol/chloroform extracted serum, so was used throughout. Sera were filtered through 


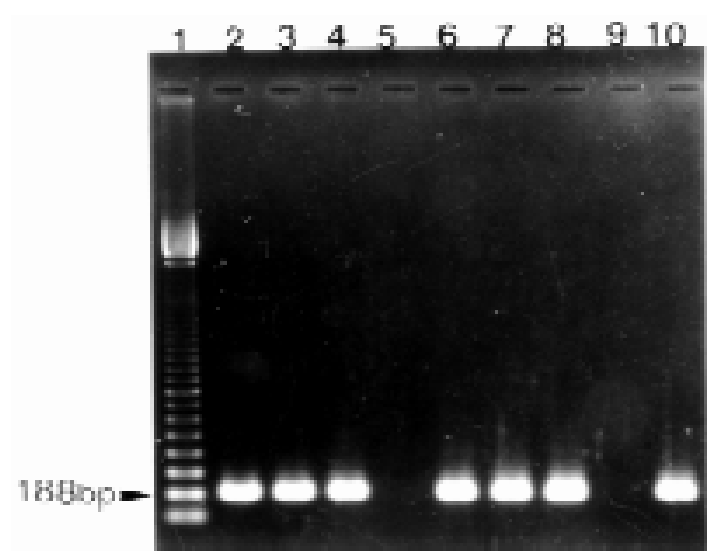

Molecular weight marker: Ladder 100 bp (lane 1), PCR based detection of Wuchereria bancrofti L3 larvae (lane 2), W. bancrofti L3 larvae added to pools of 100 (lane 3) and 50 (lane 4) whole mosquitoes, pool of 50 mosquitoes, without L3 (lane 5), patient serum obtained from "night" blood (lane 6) and "day" blood (lane 7), W. bancrofti microfilariae (lane 8 ), normal serum (lane 9), and $1 \mathrm{pg} W$. bancrofti DNA used as positive control (lane 10).

a $3.0 \mathrm{mM}$ filter in order to detect only free DNA (1 $\mathrm{ml}$ of serum was directly used for a $25 \mathrm{ml}$ PCR). Sera from individuals living in a non endemic area were used as a negative control.
Eight out of 10 sera tested, obtained from "day" or 4 out of 4 sera from "night" blood, resulted in positive PCR amplification (patient microfilariae count ranged from 50 to $1033 \mathrm{mf} / \mathrm{ml}$, as evaluated by membrane filtration). The intensity of the bands in the "day samples" and "night samples" is apparently the same (Fig. lanes 6 and 7). However, in other experiments the intensity of the bands was less in the "day samples" (not shown), what may suggest a periodicity in the concentration of free DNA in peripheral circulation.

In conclusion, these results demonstrate that PCR can detect $W$. bancrofti in C. quinquefasciatus, which may be useful for monitoring filariasis control programmes in the majority of the endemic areas, like Recife, Brazil, where C. quinquefasciatus is the vector. Finally, the detection of $W$. bancrofti free DNA makes potentially possible the diagnosis of bancroftian filariasis using blood collected during the day in areas endemic for nocturnally periodic $W$. bancrofti. Furthermore, we have shown that sera can be used in PCR without a previous DNA purification step, which reduces both costs and time.

Acknowledgements: to Dr D Freedman for critical reading of the manuscript and helpful comments. 